\title{
Generation and Dynamics of Vortex Lattices in Coherent Exciton-Polariton Fields
}

\author{
T. C. H. Liew, ${ }^{1,2}$ Yuri G. Rubo, ${ }^{1,3}$ and A. V. Kavokin ${ }^{1,4}$ \\ ${ }^{1}$ School of Physics and Astronomy, University of Southampton, Highfield, Southampton SO17 1BJ, United Kingdom \\ ${ }^{2}$ Centre for Quantum Technologies, National University of Singapore, 117543, Singapore \\ ${ }^{3}$ Centro de Investigación en Energía, Universidad Nacional Autónoma de México, Temixco, Morelos, 62580, Mexico \\ ${ }^{4}$ Marie-Curie Chair of Excellence "Polariton devices", University of Rome II, 1, via della Ricerca Scientifica, Rome, 00133, Italy
}

(Received 16 June 2008; published 31 October 2008)

\begin{abstract}
Vortex dynamics in coherent ensembles of exciton polaritons (condensates) is studied in the framework of the polarization-dependent Gross-Pitaevskii equation. Vortex lattices can be resonantly excited in the polariton field by the interference of three or more optical pumps. Vortex-antivortex pairs can also appear in polariton condensates due to scattering with disorder. The nonlinear vortex dynamics is characterized by interactions of vortex cores and vortex-antivortex recombination.
\end{abstract}

DOI: 10.1103/PhysRevLett.101.187401

PACS numbers: 78.67. $-\mathrm{n}, 42.55 . \mathrm{Sa}, 71.36 .+\mathrm{c}$

Introduction.-Recent discoveries of polariton BoseEinstein condensation (BEC) in planar semiconductor microcavities [1-3] have renewed the expectations of observing superfluidity in exciton-polariton systems [4]. One of the manifestations of the superfluid phase transition in 2D systems is the spontaneous appearance of vortex pairs at any finite temperature and their dissociation at the transition point [5]. This makes the understanding of the mechanisms of vortex formation and the dynamics of vortices in polariton systems particularly important. Interestingly, vortices may appear also in localized polariton condensates due to the effect of the static potential disorder, as recent experimental work has shown [6]. Moreover, different mechanisms of vortex creation by resonant optical excitation have been suggested $[7,8]$.

As is well known, vortices also govern the physical properties of type II superconductors. The triangular Abrikosov lattices [9] appear when a magnetic field is applied to a superconductor. In this case, each vortex carries a quantum of magnetic flux. Clearly, this effect cannot be present in polariton superfluids, since these superfluids are not charged. However, as we show in this Letter, lattices of phase and polarization vortices similar to the Abrikosov lattices can be excited in polariton fields. In contrast to the Abrikosov lattices in superconductors, which are composed of vortices with the same phase winding number, the vortex lattices formed in polariton fields contain sublattices of vortices with opposite winding numbers. This sublattice structure leads to a peculiar dynamics for polariton vortices and to attraction and recombination of vortexantivortex pairs.

We explore the possibility of creating vortex lattices by the interference of several light beams, known in classical optics [10]. Vortices or topological defects exist in linear wave systems, in general, and in optics in particular [1114]. However, contrary to the case of linear singular optics, vortices in polariton fields are affected by nonlinear spindependent polariton-polariton interactions. Using the spin- dependent Gross-Pitaevskii equations we show that the interactions stabilize the vortex lattices.

In general any topological defect in a coherent ensemble of exciton polaritons (which we call a polariton condensate) can be characterized by two winding numbers representing the change of phase and polarization of the order parameter when one circles the vortex core. The elementary topological defect in a polariton superfluid is the half vortex having semi-integer phase and polarization winding numbers [15]. We show that half vortices appear if the cavity is excited by three linearly polarized light beams incident at oblique angles or due to the disorder scattering of elliptically polarized polaritons.

Theory.-Because of the long coherence time of polaritons [16] and the fact that at low density they behave as weakly interacting bosons [1], polariton dynamics can be treated in the mean-field approximation, which leads to the Gross-Pitaevskii (GP) equation [17,18]. This equation, commonly used in the description of atomic BoseEinstein condensates [19-21], has been applied to semiconductor microcavities to describe several phenomena, including the suppression of Rayleigh scattering by impurities [17], the spatial structure of microcavity parametric oscillator polaritons [22,23], the dispersion of polariton superfluids [18], the Bose glass phase [24], and the interference of polariton condensates [25].

Polaritons have two possible spin projections on the structure growth axis, $\sigma= \pm 1$, corresponding to the right $\left(\sigma_{+}\right)$and left $\left(\sigma_{-}\right)$circular polarizations of external photons [26]. The spin-dependent GP equation is [27]:

$$
\begin{aligned}
i \hbar \frac{\partial \psi_{\sigma}}{\partial t}= & \left(\hat{H}_{\mathrm{LP}}(-i \hat{\nabla})-\frac{i \hbar}{2 \tau}+W(\mathbf{r})\right) \psi_{\sigma}+\left(\alpha_{1}\left|\psi_{\sigma}\right|^{2}\right. \\
& \left.+\alpha_{2}\left|\psi_{-\sigma}\right|^{2}\right) \psi_{\sigma}+p_{\sigma}(\mathbf{r}, t) e^{-i E_{p} t / \hbar},
\end{aligned}
$$

where the $\sigma$ polarized internal cavity polariton field, $\psi_{\sigma}$, depends on the spatial coordinate, $\mathbf{r}$. The kinetic energy operator $\hat{H}_{\mathrm{LP}}$ represents the dispersion of polaritons. We consider only lower branch polaritons from the strong 
light-matter coupling - the excitation of upper branch polariton population is negligible since we consider a pump resonant with the lower branch. $\tau$ is the polariton lifetime. $W(\mathbf{r})$ represents a potential experienced by polaritons. $\alpha_{1(2)}$ is the matrix element of polariton-polariton interactions in the parallel spin(antiparallel spin) configuration, respectively. It is well known that for 2D excitons and exciton polaritons the interactions between polaritons of parallel spin are repulsive $\left(\alpha_{1}>0\right)$. The system is thus equivalent to a self-defocusing Kerr medium in which stable optical vortex solitons have been observed [28]. The exchange interaction between polaritons dominates over the direct one, and thus polariton-polariton interactions are spindependent $\left|\alpha_{2}\right|<\alpha_{1}, \alpha_{2}<0$ [29-32]. The driving optical pump field is given by $p_{\sigma}(\mathbf{r}, t)$ and $E_{p}$ is the pump energy. The time dependence of $p_{\sigma}(\mathbf{r}, t)$ allows for a pulsed excitation, which we take as a Gaussian in time.

Vortex lattices.-We first study an ideal semiconductor microcavity without disorder $[W(\mathbf{r})=0$ everywhere $]$. As two examples, we consider the case of excitation of polaritons by three and five optical pumps in equilateral geometry (Fig. 1). The pumps are assumed to be degenerate and resonant with the lower polariton branch, all having the same magnitude of in-plane wave vector.

For simplicity we first consider the excitation by cocircularly polarized pumps. In this case only phase vortices with integer winding numbers are possible. In order to study vortex dynamics we model excitation by a 5 ps long optical pulse. Figs. 2 and 3 show the evolution of the polariton intracavity field phase (that is, the argument of $\psi_{\sigma=1}$ ) for the vortex lattices excited by three and five pumps, respectively. The phase is measured relative to the pump phase. In the linear regime (shown by left-hand columns in Figs. 2 and 3) the optical pulse initially creates a vortex lattice at $t=0 \mathrm{ps}$. In the case of a three-pump excitation, the lattice is triangular and at first glance looks similar to an Abrikosov vortex lattice in a type II superconductor. However, unlike the Abrikosov lattice it has an internal structure composed of both vortices (winding
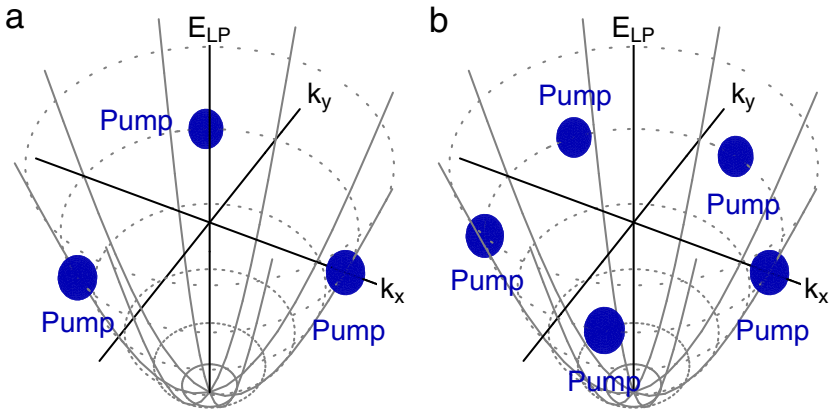

FIG. 1 (color online). Degenerate excitation of polaritons in equilateral geometries with three pumps (a) and five pumps (b). Each pump is assumed to be a Gaussian in wave vector space, centered at the positions of the spots illustrated (the Gaussian pumps are cocentered in real space). number +1 ) and antivortices (winding number -1 ). The vortex and antivortex sublattices have a honeycomb symmetry. In time, vortices and antivortices are attracted and annihilate each other due to ballistic motion of exciton polaritons in the plane of the cavity. Vortices and antivortices furthest from the pump-spot center $(x=0, y=0)$ recombine first.

The five-pump excitation allows one to create a Penroselike quasicrystal lattice [33] of vortices, which can also be decomposed into vortex and antivortex sublattices. Melting of the lattice and recombination of vortex-antivortex pairs is again apparent at later times. In the nonlinear regime (shown by right-hand columns in Figs. 2 and 3) the repulsion of polaritons caused by interactions leads to an expansion of the polariton distribution. A greater separation between vortices and antivortices is thus apparent at any given time in the nonlinear regime. This increases the lifetime of the vortices and antivortices by suppressing their recombination. Clearly, this dynamics is very different to the Abrikosov vortex dynamics. In order to evidence melting of the vortex lattices here and further we use a polariton lifetime, $\tau$, of $100 \mathrm{ps}$. This value, large compared to typical ground state polariton lifetimes in planar cavities, can be achieved in high $Q$-factor cavities for polaritons having large in-plane wave vectors at positive photonexciton detuning.

To observe half vortices [15] we now consider the excitation of a vortex lattice using three transverse electric
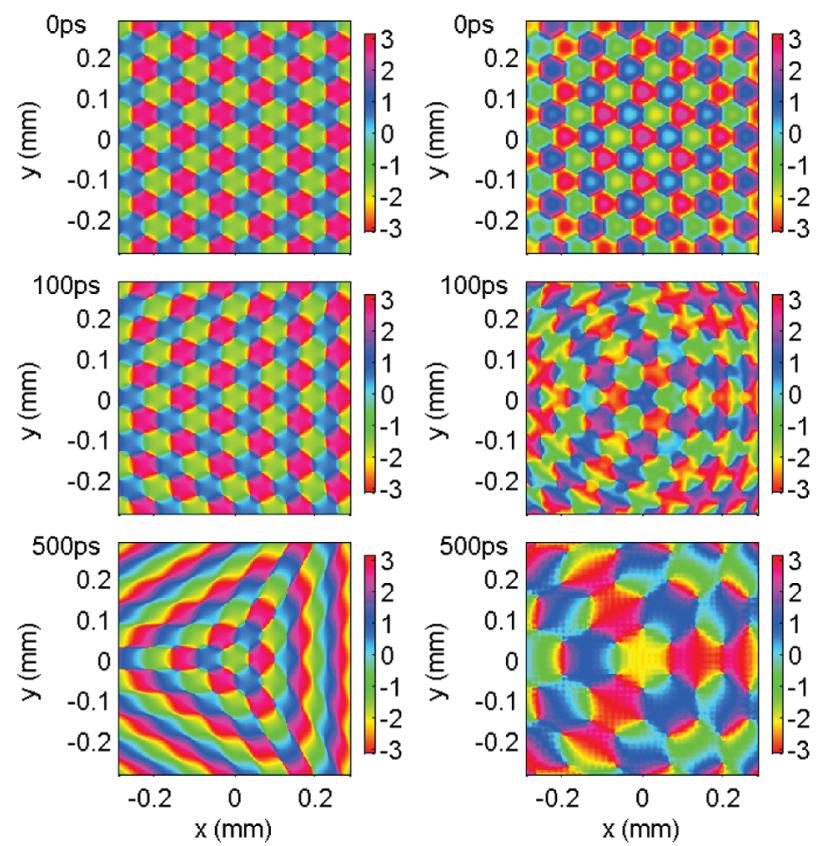

FIG. 2 (color). Evolution of the circularly polarized threepump vortex lattice phase in the linear (left) and nonlinear (right) regimes. The times indicated are relative to the pulse arrival time. Parameters: pump in-plane wave vector $=65.6 \mathrm{~mm}^{-1}$, polariton effective mass $=10^{-5} \times$ free electron mass, pump $\mathrm{FWHM}=50 \mu \mathrm{m}$. 

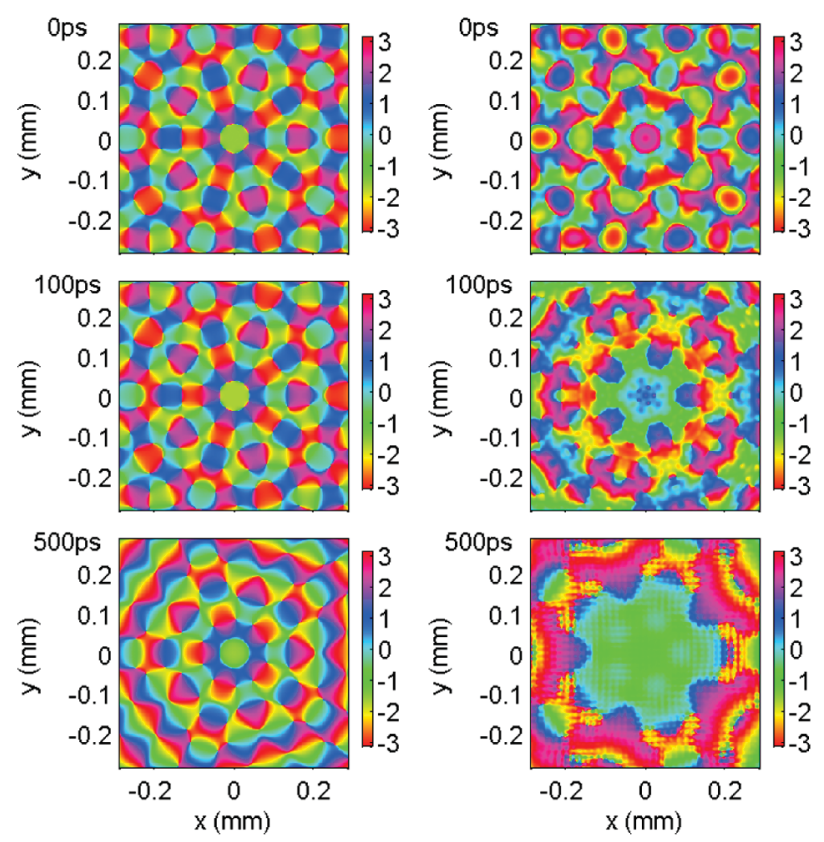

FIG. 3 (color). Evolution of the circularly polarized five-pump vortex lattice phase in the linear (left) and nonlinear (right) regimes.

(TE) polarized pumps. The results in the nonlinear regime are shown in Fig. 4. The presence of half vortices is characterized by the displacement of the phase vortex cores in the $\sigma_{+}$and $\sigma_{-}$polarized fields. In the linear regime (not shown) the recombination of vortices and antivortices in each polarization proceeds independently of the other po-
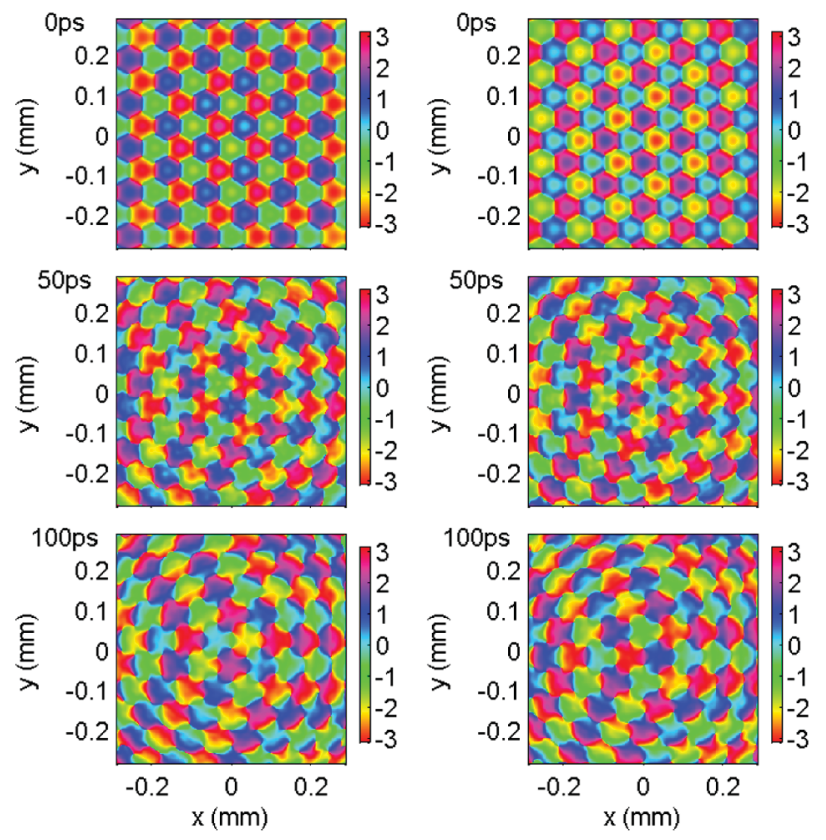

FIG. 4 (color). Evolution of the TE polarized three-pump vortex lattice phase in the nonlinear regime. Left column: $\sigma_{+}$ phase; Right column: $\sigma_{-}$phase. $\alpha_{2}=-0.1 \alpha_{1}$. larization. In the nonlinear regime the dominant repulsive interactions between cocircularly polarized polaritons causes an expansion of the polariton distribution, which increases the average distance between vortices as in the circularly polarized case.

Disorder and the natural generation of vortices.Although the simultaneous excitation of a microcavity by multiple coherent beams is experimentally feasible [25], the beam alignment can become increasingly more challenging as the number of beams increases. For this reason we would like to highlight the possibility of creating vortices using a single pump and exploiting the scattering of polaritons with disorder. The disorder in microcavities, caused by alloy and layer thickness variations in the nanostructure, can be modeled by a continuous disorder potential, $W(\mathbf{r})$ in Eq. (1), characterized by a Gaussian correlation length and root mean squared amplitude [34]. The coherent and elastic scattering of polaritons with disorder effectively creates a field of superimposed waves traveling in different directions. In the same way that a field of multiple superimposed pumps with different directions of in-plane wave vectors contains vortices, the field generated from scattering with disorder also contains vortices as shown in Fig. 5. Although we have considered here the nonlinear regime, it is important to realize that such vortices would also appear in the linear regime and are thus not related to the Berezinskii-Kosterlitz-Thouless phase transition. Indeed the generation of dislocations upon scattering from a rough surface has been evidenced in early experiments in acoustics [11] and is an interesting current topic in optics $[35,36]$. Very recently, the disorder induced phase vortices have been observed in polariton condensates [6]. The formation of half vortices, predicted to be elementary topological defects of polariton superfluids [15], can be evidenced by polarization resolved interferometry. Here, the anisotropic polariton-polariton interactions gen-
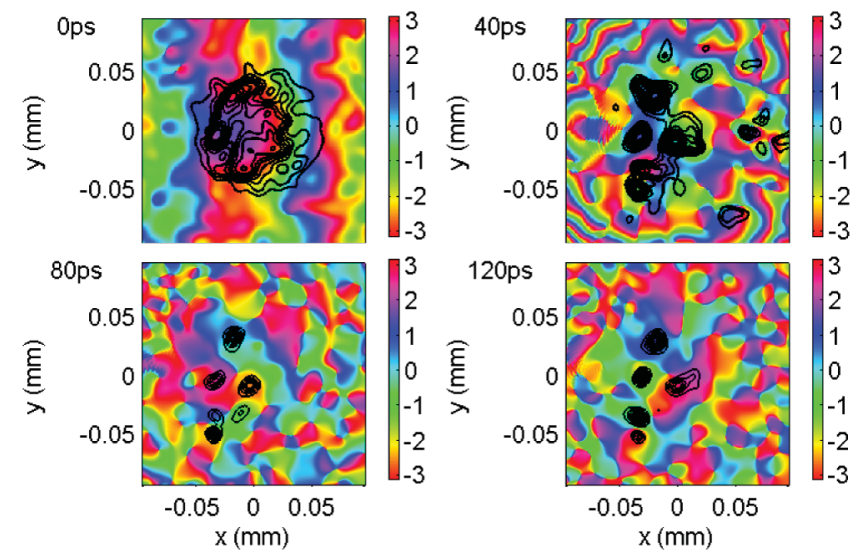

FIG. 5 (color). Evolution of the phase of the polariton field excited by a single circularly polarized pulse in a disordered microcavity (nonlinear regime). Black contours illustrate the distribution of polariton intensity. Disorder root mean squared amplitude $=0.1 \mathrm{meV}$; Gaussian correlation length $=5 \mu \mathrm{m}$. 


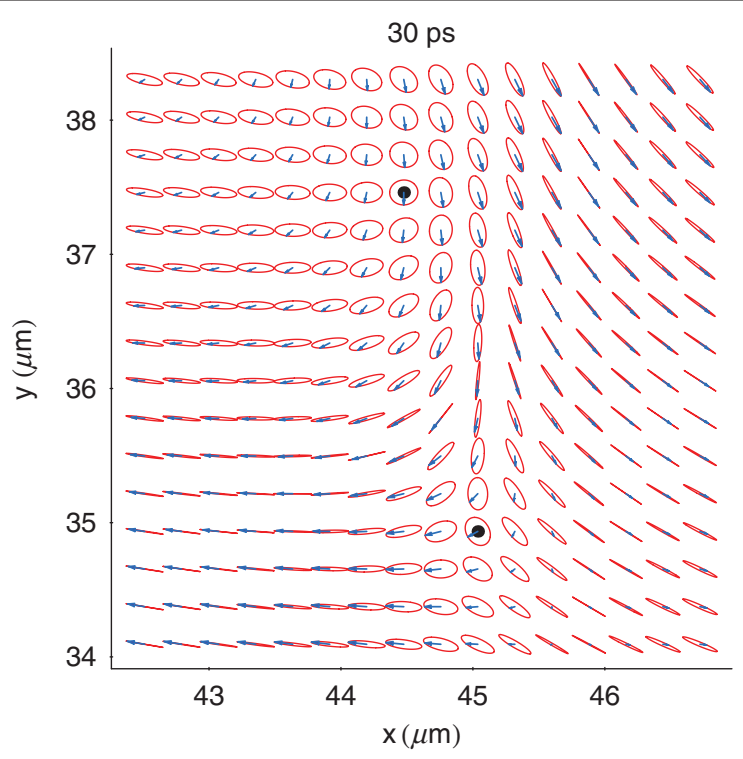

FIG. 6 (color). Polarization texture of two half vortices generated by an elliptically polarized pulse in a disordered potential. Ellipses show the path traced out by the electric field vectors (shown by radii); black spots pinpoint half vortices.

erate an anisotropy in the evolution of the $\sigma_{+}$and $\sigma_{-}$ component fields leading to a separation of vortex locations in each polarization. The polarization and phase of neighboring half vortices is shown in Fig. 6 by polarization ellipses.

Conclusion.-Coherent fields of vortices and antivortices can be excited artificially in semiconductor microcavities using multiple optical beams or via the scattering of polaritons with disorder. The elementary polariton vortices are half vortices, characterized by a change in the polarization vector as well as the phase as one circles the vortex core. Vortex dynamics can be summarized by the attraction of vortices of opposite winding number and their annihilation when they meet. The repulsive interactions between polaritons cause a spreading of a polariton distribution, which results in an associated separation of vortices and antivortices. This increased separation delays their recombination thus increasing the lifetime of a vortex lattice. The study of the elementary properties of vortices is important for the interpretation of experimental results; while the lifetime of vortices is increased in the nonlinear regime they cannot necessarily be associated with a BerezinskiiKosterlitz-Thouless phase transition. The experimental creation of a vortex lattice could also see technological application as a lattice of optical memory elements [37], if one adopts the spin-dependent bistability [27] known in semiconductor microcavities.

We thank the EPSRC for support. Y. G. R. also acknowledges DGAPA-UNAM (Grant IN107007) for support.
[1] J. Kasprzak et al., Nature (London) 443, 409 (2006).

[2] R. Balili, V. Hartwell, D. Snoke, L. Pfeiffer, and K. West, Science 316, 1007 (2007).

[3] C. W. Lai et al., Nature (London) 450, 529 (2007).

[4] A. Amo et al., arXiv:0711.1539v1.

[5] V. L. Berezinskii, Sov. Phys. JETP 32, 493 (1970); 34, 610 (1972); J. M. Kosterlitz and D. J. Thouless, J. Phys. C 6, 1181 (1973); J. M. Kosterlitz, J. Phys. C 7, 1046 (1974).

[6] K. G. Lagoudakis et al., Nature Phys. 4, 706 (2008).

[7] D. M. Whittaker, Superlattices Microstruct. 41, 297 (2007).

[8] T. C. H. Liew, A. V. Kavokin, and I. A. Shelykh, Phys. Rev. B 75, 241301 (2007).

[9] A. A. Abrikosov, Zh. Eksp. Teor. Fiz. 32, 1442 (1957) [Sov. Phys. JETP 5, 1174 (1957)].

[10] K. O'Holleran, M. J. Padgett, and M. R. Dennis, Opt. Express 14, 3039 (2006).

[11] J. F. Nye and M. V. Berry, Proc. R. Soc. A 336, 165 (1974).

[12] J. F. Nye, Natural Focusing and Fine Structure of Light (IOP Publishing, Bristol, 1999).

[13] M. S. Soskin and M. Vasnetsov, Prog. Opt. 42, 219 (2001).

[14] F. Flossmann, U. T. Schwarz, M. Maier, and M. R. Dennis, Phys. Rev. Lett. 95, 253901 (2005).

[15] Yu. G. Rubo, Phys. Rev. Lett. 99, 106401 (2007).

[16] W. Langbein et al., Phys. Rev. B 75, 075323 (2007).

[17] I. Carusotto and C. Ciuti, Phys. Rev. Lett. 93, 166401 (2004).

[18] I. A. Shelykh et al., Phys. Rev. Lett. 97, 066402 (2006).

[19] F. Dalfovo, S. Giorgini, L. P. Pitaevskii, and S. Stringari, Rev. Mod. Phys. 71, 463 (1999).

[20] A. J. Leggett, Rev. Mod. Phys. 73, 307 (2001).

[21] L. Pitaevskii and S. Stringari, Bose-Einstein Condensation (Oxford University Press, New York, 2003).

[22] D. M. Whittaker, Phys. Status Solidi C 2, 733 (2005).

[23] D. Sanvitto et al., Phys. Rev. B 73, 241308 (2006).

[24] G. Malpuech et al., Phys. Rev. Lett. 98, 206402 (2007).

[25] C. Leyder et al., Phys. Rev. Lett. 99, 196402 (2007).

[26] I. A. Shelykh, A. V. Kavokin, and G. Malpuech, Phys. Status Solidi B 242, 2271 (2005).

[27] N. A. Gippius et al., Phys. Rev. Lett. 98, 236401 (2007).

[28] G. A. Swartzlander, Jr. and C. T. Law, Phys. Rev. Lett. 69, 2503 (1992).

[29] C. Ciuti et al., Phys. Rev. B 58, 7926 (1998).

[30] P. Renucci et al., Phys. Rev. B 72, 075317 (2005).

[31] M. Combescot and O. Betbeder-Matibet, Phys. Rev. B 74, 125316 (2006).

[32] S. Schumacher, N. H. Kwong, and R. Binder, Phys. Rev. B 76, 245324 (2007).

[33] S. P. Gorkhali, J. Qi, and G. P. Crawford, J. Opt. Soc. Am. B 23, 149 (2006).

[34] V. Savona and W. Langbein, Phys. Rev. B 74, 075311 (2006).

[35] K. O'Holleran, M. R. Dennis, F. Flossmann, and M. J. Padgett, Phys. Rev. Lett. 100, 053902 (2008).

[36] F. Flossmann, K. O'Holleran, M. R. Dennis, and M. J. Padgett, Phys. Rev. Lett. 100, 203902 (2008).

[37] I. A. Shelykh, T. C. H. Liew, and A. V. Kavokin, Phys. Rev. Lett. 100, 116401 (2008). 